\title{
Resonance-assisted decay of nondispersive wave packets
}

\author{
Sandro Wimberger, ${ }^{1}$ Peter Schlagheck ${ }^{2}$ Christopher Eltschka, ${ }^{2}$ and Andreas Buchleitner ${ }^{3}$ \\ ${ }^{1}$ CNR-INFM and Dipartimento di Fisica E. Fermi, \\ Unversità degli Studi di Pisa, Largo Pontecorvo 3, 56127 Pisa, Italy \\ ${ }^{2}$ Institut für Theoretische Physik, Universität Regensburg, 93040 Regensburg, Germany \\ ${ }^{3}$ Max-Planck-Institut für Physik komplexer Systeme, Nöthnitzer Str. 38, 01187 Dresden, Germany
}

\begin{abstract}
We present a quantitative semiclassical theory for the decay of nondispersive electronic wave packets in driven, ionizing Rydberg systems. Statistically robust quantities are extracted combining resonance assisted tunneling with subsequent transport across chaotic phase space and a final ionization step.
\end{abstract}

PACS numbers: 03.65.Xp,32.80.-t,05.45.Mt,05.60.Gg

The accurate and quantitative modeling of open quantum systems at high spectral densities remains a serious challenge for quantum theory and computational physics. Even the ab initio treatment of apparently simple, atomic one or two electron systems under nonperturbative forcing, in the energy range of laboratory experiments, has become available only during the last two decades 1 ]. Furthermore, it appears a safe bet that the driven three body Coulomb problem [2] will be the most complicated "many particle" quantum problem still amenable to a (numerically) exact and complete solution, even on the most powerful supercomputers. Thus, alternative theoretical strategies which allow a precise treatment, without saturating our computational resources nor loosing quantitative predictive power, are in need.

Specifically in the range of high spectral densities, semiclassical approaches - which try to deduce the spectral structure of a given quantum system from the underlying Hamiltonian dynamics - open such an avenue. However, while semiclassics of bounded systems is well developed [3], its generalization for open systems [4] has not been fully accomplished yet: Our semiclassical understanding of tunneling and decay phenomena in systems with mixed regular-chaotic classical phase space structure remains rather incomplete, and semiclassics still has to prove its potential to come up with robust (a priori) quantitative predictions for specific experiments.

In the present Letter, we improve on that situation, by elaborating a fully quantitative semiclassical treatment of a paradigmatic example of open system dynamics at high spectral densities, in the context of light-matter interaction and coherent control: Nondispersive wave packets have been shown to be ubiquitous in periodically driven quantum systems with underlying mixed regular-chaotic phase space [5, 6, 7, 8]. They can be launched along essentially arbitrary phase space trajectories, which can be manipulated in real time [9]. In contrast to "traditional" nondispersive wave packets, which rely on the harmonicity of the spectra they are built on [10], these strongly localized eigenstates of the driven system are robust against perturbations [6, 9, 11], what is a consequence of the Kolmogorov-Arnold-Moser theorem [3].

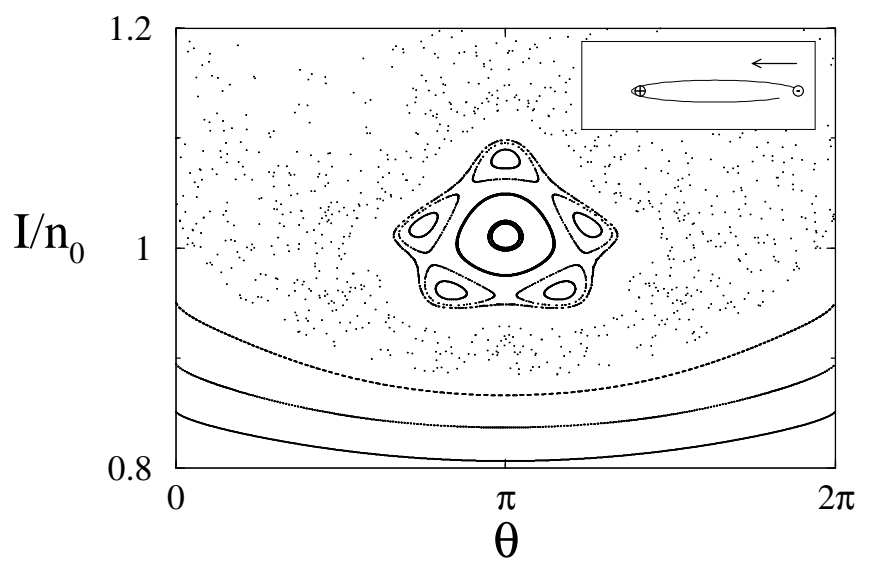

FIG. 1: Classical phase space of driven hydrogen, generated by the Hamiltonian (1), for $\omega_{0}=1$ and $F_{0}=0.041$, and spanned by the action-angle variables of the unperturbed atom, at $\omega t=0$. Nondispersive quantum wave packets are localized at the center of the principal elliptic island (concentric structure in the middle of the plot) and move along the resonantly driven Kepler orbit sketched in the inset (the arrow shows the direction of the driving force at the outer turning point), in phase with the driving field. For the chosen parameters, a prominent, higher order nonlinear $5: 1$ resonance strongly affects the tunneling coupling of the principal island's eigenstates to the surrounding chaotic sea. This resonance is expressed by the five-fold structure right within the outermost torus which confines the principal island.

The characteristic property which defines their unique potential for quantum control purposes (including quantum memory applications [12], i.e., within the framework of molecular quantum computing [13]) is their essentially eternal life time on experimentally relevant time scales 7, 14. The life time is determined by the decay rate of the wave packet from the elliptic island to which it is anchored to in classical phase space (see Fig. (1), i.e., it is essentially limited by a finite quantum mechanical tunneling rate through classically impenetrable phase space barriers [7, 14, 15].

However, while always very long, the precise value of 
the wave packet's life time is not a statistically robust quantity - it fluctuates over several orders of magnitude under tiny changes of the boundary conditions, which may be stray fields or other uncontrolled imperfections in the experiment. This sensitivity stems from the coupling of the wave packet to the chaotic component of phase space, into which it is completely embedded (see Fig. 1). We will derive a quantitative semiclassical estimate for the mean value and the variance of the wave packet's decay rate, without adjustable parameters.

For the sake of clarity, we consider a simplified (yet reliable [7]) formulation of the problem we have in mind: Atomic hydrogen initially prepared in a highly eccentric (extremal parabolic) Rydberg state aligned along the field polarization axis of a linearly polarized microwave driving field of amplitude $F$ and frequency $\omega$ can be described by the Hamiltonian

$$
H\left(p_{z}, z\right)=\frac{p_{z}^{2}}{2}-\frac{1}{z}+F z \cos (\omega t),
$$

where the configuration space of the electron has been confined to the $z$-axis (for the actual three dimensional problem, such confinement can be achieved by applying a weak additional static electric field along the polarization axis of the periodic drive 7] ). The classical phase space structure generated by this Hamiltonian is illustrated in Fig. 1] and is completely fixed by the choice of the scaled driving field frequency $\omega_{0}=\omega n_{0}^{3}$ and amplitude $F_{0}=F n_{0}^{4}$, respectively, as a consequence of the scale invariance of Hamilton's equations of motion. Specifically, we set $\omega_{0}=1$ and $F_{0}=0.041$, in order to realize the essential ingredients of our subsequent treatment: (i) an elliptic island (at the center of Fig. (1), which is (ii) completely embedded into the chaotic sea, and (iii) encloses a prominent, higher order $r: s$ nonlinear resonance right within its outermost confining torus.

For different values of $n_{0}$, and accordingly adjusted $\omega$ and $F$, we find (by exact numerical diagonalization of the Floquet Hamiltonian defined by (11) 7]) wave packet eigenstates localized on the elliptic island, together with their decay rates $\Gamma_{\mathrm{wp}}$. Under changes of $n_{0}$, the latter (which, in this specific physical realization, are nothing but ionization rates mediated by multiphoton coupling to the atomic continuum) fluctuate wildly, as shown in Fig. 2 below.

To determine the mean value and the variance of $\Gamma_{\mathrm{wp}}$, let us start from the random matrix ansatz for an effective Hamiltonian employed in [15]:

$$
H_{\mathrm{eff}}=\left(\begin{array}{ccccc}
E_{\mathrm{wp}} & V_{\mathrm{rc}} & 0 & \cdots & 0 \\
V_{\mathrm{rc}} & H_{11} & \cdots & \cdots & H_{1 N} \\
0 & \vdots & & & \vdots \\
\vdots & H_{N 1} & \cdots & \cdots & H_{N N}-\frac{\mathrm{i}}{2} \Gamma_{c}
\end{array}\right) .
$$

The matrix structure comprises the wave packet state localized at the center of the regular island (with en- ergy $E_{\mathrm{wp}}$ ), and $N$ additional states located in the chaotic phase space region, one of which being subject to decay with the rate $\Gamma_{c}$. The chaotic states are assumed to be strongly coupled to each other, such that the corresponding sub-block $\left(H_{i j}\right)$ can be described by a random matrix from the Gaussian orthogonal ensemble [16]. In accordance with the resonance-assisted coupling mechanism to be explained below, we assume that the wave packet is dominantly coupled to only one of those chaotic states, via the (comparatively small) matrix element $V_{\mathrm{rc}}$. As a qualitative, conceptional improvement over [15], where $V_{\mathrm{rc}}$ and $\Gamma_{c}$ were free fitting parameters, we here derive quantitative estimates for these two coupling constants.

Within our semiclassical framework, transport to the continuum is only possible by traversing a large part of chaotic phase space. Quantum mechanically, this is equivalent to multiphoton transition amplitudes which couple the initial state - the wave packet - to Rydberg states with ionization potentials smaller than $\omega$, i.e., with principal quantum numbers $n$ above $n_{c}=n_{0}^{3 / 2} / \sqrt{2 \omega_{0}}$. The final, ionizing step occurs from these highly excited states by a one-photon process. In the effective Hamiltonian of (2), this final ionization process is accounted for by attributing a finite decay rate $\Gamma_{c}$ to one of the chaotic states, which is well estimated by the Golden Rule expression

$$
\Gamma_{c}=0.265 F^{2} \omega^{-10 / 3} n_{c}^{-3} \simeq 1.26 \times 10^{-3} n_{0}^{-5 / 2} \text { a.u. }
$$

for the single-photon ionization rate of a Rydberg state with quantum number $n_{c}$ [17.

A random-matrix average over the eigenvalues and eigenvectors of the chaos block $\left(H_{i j}\right)$ gives rise to the Cauchy-type probability density

$$
P\left(\Gamma_{\mathrm{wp}}\right)=\frac{1}{\pi} \frac{\sqrt{\Gamma_{0} / \Gamma_{\mathrm{wp}}}}{\Gamma_{\mathrm{wp}}+\Gamma_{0}}
$$

of the wave packet's decay rate 15. The characteristic scale $\Gamma_{0}$ is derived as $\Gamma_{0}=\left(\pi V_{\mathrm{rc}} / \omega\right)^{2} \Gamma_{c}$, exploiting that the chaotic eigenvalues are uniformly distributed within an energy interval of width $\omega$, due to the inherent periodicity of the Floquet spectrum. Because of the large fluctuations implied by the distribution (4), the relevant statistical quantity is given by the averaged logarithm [18]. An explicit calculation of this geometric mean gives

$$
\bar{\Gamma}_{\mathrm{wp}} \equiv \exp \left[\left\langle\ln \left(\Gamma_{\mathrm{wp}}\right)\right\rangle\right]=\Gamma_{0}=\left(\frac{\pi V_{\mathrm{rc}}}{\omega}\right)^{2} \Gamma_{c},
$$

which depends only on the single-photon ionization rate $\Gamma_{c}$ from (3), on the driving frequency $\omega$, and on the coupling matrix element $V_{\mathrm{rc}}$ between the regular island and its chaotic surrounding. $V_{\mathrm{rc}}$ can be derived using purely semiclassical arguments, as follows.

Let us recall that nonlinear resonances between the external drive and the local modes of a regular phase 
space region induce higher order perturbative couplings between the locally quantized eigenstates of that region 19. This leads to the phenomenon of resonance-assisted tunneling which, originally proposed for near-integrable dynamics, can be generalized to mixed regular-chaotic systems as well [20]. There it provides the dominant semiclassical mechanism for the tunneling process that connects the "ground state" of a regular island (localized at the island's center) to states within the chaotic sea (which, in turn, are coupled to the atomic continuum with a rate $\Gamma_{c}$, given in Eq. (3)).

Quantitatively, the classical dynamics near a $r: s$ resonance ( $s$ oscillations match $r$ driving periods) is described by the effective pendulum Hamiltonian 19]

$$
H_{\mathrm{res}}=\frac{\left(\widetilde{I}-I_{r: s}\right)^{2}}{2 m_{r: s}}+2 V_{r: s} \cos (r \widetilde{\theta}),
$$

expressed in terms of the local action-angle variables $\widetilde{I}, \widetilde{\theta}$ of the principal elliptic island, where $s$ is absorbed in the definition of $\widetilde{\theta}$. The central action $I_{r: s}$ of the $r: s$ resonance, and the effective mass and coupling parameters $m_{r: s}, V_{r: s}$, can be extracted with little numerical effort from the classical phase space, by computing the area covered by the separatrices of the $r: s$ resonance, and by evaluating the stability matrix of the associated periodic points [20]. Quantizing (6), we see that the perturbation term induced by the $r: s$ resonance couples the $k$ th excited state of the principal island, given by the plane wave $\langle\widetilde{\theta} \mid k\rangle \sim \exp (i k \widetilde{\theta})$ in angle space, to the states $|k \pm r\rangle$, with a strength $V_{r: s}$. Since the wave packet state corresponds to the principal island's ground state with $k=0$, its coupling to the chaotic sea is thus given by the effective matrix element

$$
V_{\mathrm{rc}}=V_{r: s} \prod_{l=1}^{l_{c}-1} \frac{V_{r: s}}{\widetilde{E}_{0}-\widetilde{E}_{l \cdot r}},
$$

with $\widetilde{E}_{k}=\left(\widetilde{I}_{k}-I_{r: s}\right)^{2} /\left(2 m_{r: s}\right)$ and $\widetilde{I}_{k}=\hbar(k+1 / 2)$. Here, $\left|l_{c} \cdot r\right\rangle$ denotes the first state within this perturbative sequence of higher order couplings that is located outside the principal island, and the prefactor $V_{r: s}$ accounts for the final step from $\left|\left(l_{c}-1\right) \cdot r\right\rangle$ to $\left|l_{c} \cdot r\right\rangle$.

In our exemplary case of Fig. 1] the driving induces a prominent 5:1 resonance within the principal regular island on which the wave packet is localized. From the area covered by the principal island - which is determined numerically - we deduce $n_{0} \simeq 135$ as the critical quantum number at which the island's fifth excited state (i.e., the first state to which the ground state is coupled by the $5: 1$ resonance) is located exactly on the outermost invariant torus. For $n_{0}<135$, we can therefore identify this fifth excited state with the first basis state of the chaos block in the Hamiltonian (2), and set $V_{\mathrm{rc}}=V_{r: s}$, which, together with Eqs. (35) and the numerically computed value of $V_{r: s}$, leads to our central result

$$
\bar{\Gamma}_{\mathrm{wp}} \simeq 9.6 \times 10^{-13} n_{0}^{-1 / 2} \text { a.u. }, n_{0}<135,
$$

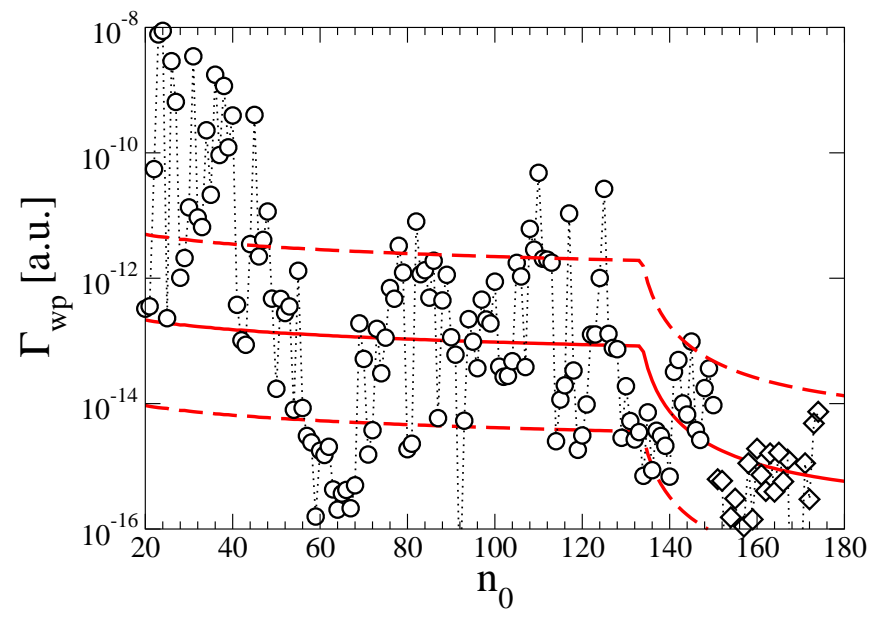

FIG. 2: (color online). Comparison of the exact quantum decay rates of the wave packet states (symbols) with the semiclassical prediction for their mean value $\bar{\Gamma}_{\text {wp }}$ [solid line, given by Eqs. (8) and (9)] and the standard deviation [dashed lines, see Eq. [10] ]. The rates are plotted as a function of the principal quantum number $n_{0}$ of the Rydberg orbit along which the wave packet is launched. The quantum results were obtained for the same field parameters as Fig. 1] Open circles and diamonds distinguish different basis sizes form the numerical diagonalization. For $n_{0} \leq 150$, the basis was chosen such as to resolve all bound states with an ionization potential larger and equal to the one-photon energy $\omega$. For $n_{0}>150$, only bound states below $2 n_{0}$ were numerically resolved.

for the mean decay rate of the wave packet. At larger principal quantum numbers $\left(n_{0}>135\right)$, the coupling from the regular island to the chaotic sea involves one more perturbative step in Eq. (7), which substantially reduces the matrix element $V_{\mathrm{rc}}$. This leads to

$$
\bar{\Gamma}_{\mathrm{wp}} \simeq 1.7 \times 10^{-20} \frac{n_{0}^{7 / 2}}{\left(n_{0}-131\right)^{2}} \text { a.u. }, n_{0}>135 .
$$

As a consequence, the semiclassically calculated decay rates exhibit a sudden drop at $n_{0} \simeq 135$. (9) describes the monotonic decrease (mind the expression's denominator!) of the rates with $n_{0}$ up to $n_{0} \simeq 250$, where an additional drop is expected to occur by virtue of yet another perturbative step in Eq. (7).

Fig. 2 highlights that our semiclassical result $\bar{\Gamma}_{\text {wp }}$ fits the exact quantum decay rates $\Gamma_{\mathrm{wp}}$ of the wave packet very well. On average, after a first exponential decrease for $n_{0} \leq 60$, the decay rate settles to a plateau at $\bar{\Gamma}_{\mathrm{wp}} \simeq 10^{-13}$ a.u., and eventually drops again around $n_{0} \geq 135$. Beyond that, our model also succeeds to very well characterize the quantum rates' fluctuations by their logarithmic standard deviation derived from (4):

$$
\sqrt{\operatorname{var}\left[\ln \left(\Gamma_{\mathrm{wp}}\right)\right]}=\sqrt{\left\langle\left[\ln \left(\Gamma_{\mathrm{wp}}\right)-\ln \left(\bar{\Gamma}_{\mathrm{wp}}\right)\right]^{2}\right\rangle}=\pi .
$$

This universal result is indicated by the dashed lines in Fig. 2] which are given by $\bar{\Gamma}_{\mathrm{wp}} e^{ \pm \pi}$ and which encompass 
the overwhelming part of the data. Despite the sensitive dependence of individual decay rates on boundary conditions and classical phase space structures, our combined semiclassical and random matrix approach provides a robust statistical characterization, over a wide range of excitation energies.

The disagreement between our semiclassical result and the quantum data below $n_{0} \simeq 60$ is not surprising, since, at such low excitations, the principal island cannot accommodate a sufficient number of quantum eigenstates to resolve the 5 : 1 resonance (approx. $r / 2$ states are needed for satisfactory resolution [20]). Thus, a necessary condition (item (iii) above) for the resonance assisted tunneling mechanism to manifest itself is not satisfied at too small $n_{0} \lesssim 60$.

In conclusion, the plateau-like structure born out in Fig. 2 in the range $n_{0} \simeq 60 \ldots 135$, corroborates the relevance of the resonance assisted tunneling mechanism for the decay properties of nondispersive wave packets, in an experimentally routinely accessible energy range [5, 9]. Note that the average trend $\Gamma_{\text {wp }} \sim n_{0}^{-1 / 2}$, predicted by (8) and confirmed in Fig. 2] is markedly different from an on average exponential decay with $n_{0}$, as assumed in [15, 21]. Indeed, a similar step structure arises in earlier published data on nondispersive hydrogen wave packets 21] (at a slightly different field amplitude), which, on the basis of our presently improved understanding, is also attributed to resonance-assisted tunneling. Thus, while the precise extension of the plateau depends on the specific choice of parameter values, and certainly also on the effective dimensionality of the electron's configuration space, the structure as such is a robust fingerprint of resonance assisted tunneling, as well as the universal value $\pi$ of the decay rates' standard deviation. An experimental verification of this prediction is challenging, though appears in reach for the most advanced experimental setups to date [5, 9].

We thank Italo Guarneri for inspiring discussions, and acknowledge support by the Alexander von Humboldt Foundation and the DFG.

[1] C. Iu et al., Phys. Rev. Lett. 66, 145 (1991); R. Püttner et al., ibid. 86, 3747 (2001); A. Krug and A. Buchleitner, ibid. 86, 3538 (2001); J. Madroñero and A. Buchleitner, ibid. 95, 263601 (2005).

[2] A. Scrinzi and B. Piraux, Phys. Rev. A 56, R13 (1997);
L. Nikolopoulos and P. Lambropoulos, J. Phys. B 34, 545 (2001); J. S. Parker, B. J. S. Doherty, K. J. Meharg, and K. T. Taylor, ibid. 36, L393 (2003); B. Piraux, J. Bauer, S. Laulan, and H. Bachau, Eur. Phys. J. D 26, 7 (2003); J. Madroñero and A. Buchleitner, in High Performance Computing in Science and Engineering, Munich 2004, S. Wagner et al. eds. (Springer, Berlin, 2004), p. 329 ; C. Ruiz, L. Plaja, L. Roso, and A. Becker, Phys. Rev. Lett. 96, 053001 (2006).

[3] A. M. Ozorio de Almeida, Hamiltonian Systems: Chaos and Quantization (Cambridge University Press, Camebridge, 1988).

[4] I. Garcia-Mata and M. Saraceno, Phys. Rev. E 69, 056211 (2004).

[5] D. Arbó et al., Phys. Rev. A 67, 063401 (2003).

[6] S. Wimberger, I. Guarneri, and S. Fishman, Nonlinearity 16, 1381 (2003).

[7] A. Buchleitner, D. Delande, and J. Zakrzewski, Phys. Rep. 368, 409 (2002).

[8] I. Białynicki-Birula, M. Kalinski, and J. H. Eberly, Phys. Rev. Lett. 73, 1777 (1994); E. Lee, A. F. Brunello, and D. Farrelly, ibid. 75, 3641 (1995); S. Fishman, I. Guarneri, and L. Rebuzzini, ibid. 89, 084101 (2002); J. Ortigoso, ibid. 93, 073001 (2004); M. Kalinski, L. Hansen, and D. Farrelly, Phys. Rev. Lett. 95, 103001 (2005).

[9] H. Maeda, D. Norum, and T. F. Gallagher, Science 307, 1757 (2005).

[10] C. Raman, T. Weinacht, and P. Bucksbaum, Phys. Rev. A 55, R3995 (1997).

[11] H. Maeda and T. F. Gallagher, Phys. Rev. Lett. 92, 133004 (2004).

[12] J. Ahn, T. Weinacht, and P. Bucksbaum, Science 287, $463(2000)$.

[13] C. M. Tesch and R. de Vivie-Riedle, Phys. Rev. Lett. 89, 157901 (2002).

[14] D. Delande and A. Buchleitner, Adv. At. Mol. Opt. Phys. 34, 85 (1994).

[15] J. Zakrzewski, D. Delande, and A. Buchleitner, Phys. Rev. E 57, 1458 (1998).

[16] S. Tomsovic and D. Ullmo, Phys. Rev. E 50, 145 (1994).

[17] G. Casati, I. Guarneri, and D. L. Shepelyansky, IEEE J. Quantum Electron. 24, 1420 (1988).

[18] B. Kramer and A. MacKinnon, Rep. Prog. Phys. 56, 1469 (1993).

[19] A. M. Ozorio de Almeida, J. Phys. Chem. 88, 6139 (1984); L. Bonci, A. Farusi, P. Grigolini, and R. Roncaglia, Phys. Rev. E 58, 5689 (1998); O. Brodier, P. Schlagheck, and D. Ullmo, Phys. Rev. Lett. 87, 064101 (2001); S. Keshavamurthy, Phys. Rev. E 72, 045203(R) (2005).

[20] C. Eltschka and P. Schlagheck, Phys. Rev. Lett. 94, 014101 (2005).

[21] K. Hornberger and A. Buchleitner, Europhys. Lett. 41, 383 (1998). 